\title{
Perturbative Approach to Calculating the Correlation Function of bi-isotropic Metamaterials
}

\author{
Marjan Jafari* and Fateme Moradi \\ Department of Physics, International University of Imam Khomeini, Qazvin, Iran \\ *Corresponding Author's Email: jafary.marjan@gmail.com
}

Received: Aug. 7, 2017, Revised: Oct. 15, 2017, Accepted: Nov. 11, 2017, Available Online: Dec. 1, 2018

DOI: 10.29252/ijop.12.2.137

\begin{abstract}
A bi-isotropic magneto-electric metamaterials is modeled by two independent reservoirs. The reservoirs contain a continuum of three dimensional harmonic oscillators, which describe polarizability and magnetizability of the medium. The paper aimed to investigate the effect of electromagnetic field on bi-isotropic. Starting with a total Lagrangian and using EulerLagrange equation, researcher could obtain a quantum Langevin type dissipative equation for electromagnetic field. Generating functional of the system is obtained by the path integral method and based on the perturbative approach. By generating functional, a series expansion in terms of susceptibility function of the bi-isotropic metamaterials is obtained for correlation function or two-point Green's function. In special case, the close relationship between statistical mechanics and quantum field theory,which was reflected in the path integral methods, could obtain free energy of electromagnetic field for isotropic metamaterial using two-point Green's function. As an example, the Casimir force of two polarizable metamaterial spheres by Lorentz susceptibilities was studied. Furthermore, Casimir force of two polarizable-magnetizable metamaterials was calculated.
\end{abstract}

KEYWORDS: Bi-isotropic, Casimir force, Correlation function, Generation functional, Metamaterials.

\section{INTRODUCTION}

Quantum field theory is the quantum mechanics of continuous system which is fully developed in quantum electrodynamics. This theory is considered as the most successful theory in physics. It is obvious that the quantum properties of electromagnetic field can be influenced by the presence of media [13]. Complex materials and metamaterials have played a pivotal role in the presence of electromagnetic fields. One of the properties that carry media into these classes is magnetoelectric coupling in materials. Such media are called bi-isotropic metamaterials [4,5].

In this paper, using path integral methods, we have focused on examining bi-isotropic electromagnetic metamaterials, which have been quantized in the presence of the electromagnetic field. There are many approaches to quantize electromagnetic field in the presence of magneto-dielectric materials [6,7]. One approach is the path integral method. Path integrals in quantum electrodynamic field theory play a much more important role due to several reasons. They provide Green's functions with an easy road to quantization and expressions, which are related to many physical quantities [8-10]. In addition, the close relationship between statistical mechanics and quantum field theory is reflected in the path integral methods. Therefore, in quantum optics there are some problems that require us to quantize the electromagnetic field by the path integral methods such as calculating the Casimir force $[11,12]$.

The Casimir energy is the difference between the energy of the fluctuating field when the objects are present compared to their removal to infinity $[13,14]$. 
In the present work, path integrals is used to quantize the electromagnetic field in the presence of bi-isotropic metamaterials and obtain the two point correlation function of the electromagnetic field for the first time. In special case, the Casimir energy of electric metamaterials in Drude-Lorantz regime is calculated using two point correlation function.

In this method, the medium is modeled by two independent reservoirs. Each reservoir contains a continuum of three dimensional harmonic oscillators and the reservoirs describe the polarizability and magnetizability of the medium $[15,16]$.

We start from Lagrangian of the system which consists of electromagnetic field in the presence of bi-isotropic metamaterial and obtain an expansion for the two-point correlation function in terms of the electric and magnetic susceptibility function of the medium for electromagnetic field. As examples of applications of these expansions, we obtain a series expansion of the Casimir force in the presence of polarizable metamaterial in Droude-Lorentz regime and polarizable-magnetizable metamaterials.

\section{ELECTROMAGNETIC FIELD}

In this section we introduce Lagrangian of the system which consists of electromagnetic field in the presence of bi-isotropic metamaterials. In this approach, the bi-isotropic material is modeled by two continuum collection of three dimensional harmonic oscillators. Therefore, the total Lagrangian of the system is as follows

$$
L=L_{E M}+L_{m}+L_{\mathrm{int}}
$$

First term in the relation is the electromagnetic field Lagrangian

$$
L_{E M}=\frac{1}{2} \varepsilon_{0} \mathbf{E}^{2}(\mathbf{x}, t)-\frac{\mathbf{B}^{2}(\mathbf{x}, t)}{2 \mu_{0}}
$$

where $\mathbf{E}=-\partial \mathbf{A} / \partial t$ and $\mathbf{B}=\nabla \times \mathbf{A}$ represent electric and magnetic field respectively. The second term in Eq. (1) is lagrangian of the medium and the dynamical variables of the harmonic oscillator labeled by the continuous parameter $\omega$

$$
\begin{aligned}
L_{m}= & \frac{1}{2} \int d \omega\left(\dot{\mathbf{X}}_{\omega}^{2}(\mathbf{x}, \mathrm{t})-\omega^{2} \mathbf{X}_{\omega}^{2}(\mathbf{x}, \mathrm{t})\right)+ \\
& \frac{1}{2} \int d \omega\left(\dot{\mathbf{Y}}_{\omega}^{2}(\mathbf{x}, \mathrm{t})-\omega^{2} \mathbf{Y}_{\omega}^{2}(\mathbf{x}, \mathrm{t})\right)
\end{aligned}
$$

The last term in Eq. (1), $L_{i n t}$, is describing the interaction between the bi-isotropic magnetodielectric metamaterials and the electromagnetic field which is:

$$
\begin{aligned}
L_{\mathrm{int}}= & \int d \omega f_{1}(\mathbf{x}, \omega) \dot{\mathbf{A}}(\mathbf{x}, t) \cdot \mathbf{X}_{\omega}(\mathbf{x}, t)+ \\
& \int d \omega f_{2}(\mathbf{x}, t) \dot{\mathbf{A}}(\mathbf{x}, t) \cdot \mathbf{Y}_{\omega}(\mathbf{x}, t)+ \\
& \int d \omega g_{1}(\mathbf{x}, \omega) \nabla \times \mathbf{A}(\mathbf{x}, t) \cdot \mathbf{X}_{\omega}(\mathbf{x}, t)+ \\
& \int d \omega g_{2}(\mathbf{x}, \omega) \nabla \times \mathbf{A}(\mathbf{x}, t) \cdot \mathbf{Y}_{\omega}(\mathbf{x}, t)
\end{aligned}
$$

where $f_{i}, g_{i}$ and $i=1,2$ are the coupling function between the electromagnetic field and the medium [18]. We can quantize the system using path integral techniques by the Lagrangian [19]. An important quantity in the field theory is the generating functional from which n-point correlation functions is obtained. Our purpose is to find two-point correlation functions or Green's function in terms of susceptibility of the bi-isotropic medium.

Using Gaussian Integrals, the free generating function is found as follows:

$$
\begin{aligned}
W_{0}= & N \exp \left[\frac { - 1 } { 2 \hbar ^ { 2 } } \int d ^ { 4 } x \int d ^ { 4 } x ^ { \prime } \left[J_{i} G_{i j}^{0}\left(x-x^{\prime}\right) J_{j}\right.\right. \\
& +\int d \omega J_{i, \omega} G_{\omega}^{0}\left(x-x^{\prime}\right) J_{j, \omega} \delta_{i j} \\
& \left.\left.+\int d \omega J^{\prime}{ }_{i, \omega} G_{\omega}^{0}\left(x-x^{\prime}\right) J^{\prime}{ }_{j, \omega} \delta_{i j}\right]\right]
\end{aligned}
$$

where $J_{i}$ indicates the presence of the perturbation. $G_{\omega}^{0}$ and $G_{i j}^{0}$ are free Green's function of the reservoirs and electromagnetic fields [8], 


$$
\begin{aligned}
\mathbf{G}_{\omega}^{0}\left(\mathbf{x}-\mathbf{x}^{\prime}\right)=\frac{1}{4 \pi} \frac{\exp \left(-i \omega\left|\mathbf{x}-\mathbf{x}^{\prime}\right|\right)}{\left|\mathbf{x}-\mathbf{x}^{\prime}\right|} \\
\mathbf{G}_{i j}^{0}\left(\mathbf{r}=\mathbf{x}-\mathbf{x}^{\prime}, \omega\right)=-\frac{\omega^{2}}{c^{2}} \frac{\exp \left(-i \frac{\omega r}{c}\right)}{4 \pi \mathbf{r}}\left[\delta _ { i j } \left(1+i \frac{c}{\omega \mathbf{r}}-(6)\right.\right. \\
\left.\left.\frac{c^{2}}{\omega^{2} \mathbf{r}^{2}}\right)-\frac{\mathbf{r}_{i} \mathbf{r}_{j}}{\mathbf{r}^{2}}\left(1+i \frac{3 c}{\omega \mathbf{r}}-\frac{3 c^{2}}{\omega^{2} \mathbf{r}^{2}}\right)\right]+\frac{1}{3} \delta_{i j} \delta^{3}(\mathbf{r})
\end{aligned}
$$

The interacting generating functional is achieved from the free generating functional using perturbative methods as follows

$$
\begin{gathered}
W=\exp \left[\frac { i } { \hbar } \int d ^ { 4 } x \left[\int d \omega f_{1}(\mathbf{x}, \omega) \partial_{t}\left(\frac{\hbar}{i} \frac{\delta}{\delta \mathbf{J}_{0}}\right) \cdot\left(\frac{\hbar}{i} \frac{\delta}{\delta \mathbf{J}_{\omega}}\right)+\right.\right. \\
\int d \omega f_{2}(\mathbf{x}, \omega) \partial_{t}\left(\frac{\hbar}{i} \frac{\delta}{\delta \mathbf{J}_{0}}\right) \cdot\left(\frac{\hbar}{i} \frac{\delta}{\delta \mathbf{J}_{\omega}^{\prime}}\right)+ \\
\int d \omega g_{1}(\mathbf{x}, \omega) \nabla \times\left(\frac{\hbar}{i} \frac{\delta}{\delta \mathbf{J}_{0}}\right) \cdot\left(\frac{\hbar}{i} \frac{\delta}{\delta \mathbf{J}_{\omega}}\right)+ \\
\left.\left.\int d \omega g_{2}(\mathbf{x}, \omega) \nabla \times\left(\frac{\hbar}{i} \frac{\delta}{\delta \mathbf{J}_{0}}\right) \cdot\left(\frac{\hbar}{i} \frac{\delta}{\delta \mathbf{J}_{\omega}^{\prime}}\right)\right]\right] W_{0}\left[\mathbf{J}_{0}, \mathbf{J}_{\omega}, \mathbf{J}_{\omega}^{\prime}\right]
\end{gathered}
$$

The two-point correlation function is obtained by generating functional as follows

$$
\mathbf{G}_{i j}\left(x, x^{\prime}\right)=\left.(-i \hbar) \frac{\delta^{2} W\left[\mathbf{J}, \mathbf{J}_{\omega}, \mathbf{J}_{\omega}^{\prime}\right]}{\delta \mathbf{J}_{i}(x) \delta \mathbf{J}_{j}\left(x^{\prime}\right)}\right|_{\mathbf{J}=\mathbf{J}_{\omega}=\mathbf{J}_{\omega}^{\prime}=0}
$$

Using the Eq. (7) and some straightforward calculations, the following expansion for the electromagnetic Green's function dyadic in frequency variable is obtained as:

$$
\begin{aligned}
& \mathbf{G}_{E M-b i}\left(\mathbf{x}-\mathbf{x}^{\prime}, \omega\right)=\mathbf{G}^{0}\left(\mathbf{x}-\mathbf{x}^{\prime}, \omega\right)+ \\
& \int d^{3} \mathbf{x}_{1} \mathbf{G}^{0}\left(\mathbf{x}-\mathbf{x}_{1}, \omega\right) \omega^{2} \chi_{e}\left(\omega, \mathbf{x}_{1}\right) \mathbf{G}^{0}\left(\mathbf{x}_{1}-\mathbf{x}^{\prime}, \omega\right)+ \\
& \int d^{3} \mathbf{x}_{2} \int d^{3} \mathbf{x}_{1} \mathbf{G}^{0}\left(\mathbf{x}-\mathbf{x}_{1}, \omega\right) \omega^{2} \chi_{e}\left(\omega, \mathbf{x}_{1}\right)+ \\
& \mathbf{G}^{0}\left(\mathbf{x}_{1}-\mathbf{x}_{2}, \omega\right) \omega^{2} \chi_{e}\left(\omega, \mathbf{x}_{2}\right) \mathbf{G}^{0}\left(\mathbf{x}_{2}-\mathbf{x}^{\prime}, \omega\right)+\ldots \\
& \int d^{3} \mathbf{x}_{1} \nabla_{1} \times \mathbf{G}^{0}\left(\mathbf{x}-\mathbf{x}_{1}, \omega\right) \chi_{m}\left(\omega, \mathbf{x}_{1}\right) \nabla_{1} \times \mathbf{G}^{0}\left(\mathbf{x}_{1}-\mathbf{x}^{\prime}, \omega\right)+ \\
& \int d^{3} \mathbf{x}_{2} \int d^{3} \mathbf{x}_{1} \nabla_{1} \times \mathbf{G}^{0}\left(\mathbf{x}-\mathbf{x}_{1}, \omega\right) \chi_{m}\left(\omega, \mathbf{x}_{1}\right) \nabla_{1} \times \nabla_{2} \times \\
& \mathbf{G}^{0}\left(\mathbf{x}_{1}-\mathbf{x}_{2}, \omega\right) \chi_{m}\left(\omega, \mathbf{x}_{2}\right) \nabla_{2} \times \mathbf{G}^{0}\left(\mathbf{x}_{2}-\mathbf{x}^{\prime}, \omega\right)+\ldots+ \\
& 2 \int d^{3} \mathbf{x}_{1} \nabla_{1} \times \mathbf{G}^{0}\left(\mathbf{x}-\mathbf{x}_{1}, \omega\right) i \omega \chi_{e m}\left(\omega, \mathbf{x}_{1}\right) \mathbf{G}^{0}\left(\mathbf{x}_{1}-\mathbf{x}^{\prime}, \omega\right)+ \\
& 2 \int d^{3} \mathbf{x}_{2} \int d^{3} \mathbf{x}_{1} \mathbf{G}^{0}\left(\mathbf{x}-\mathbf{x}_{1}, \omega\right) \omega^{2}\left[\chi_{e}\left(\mathbf{x}_{1}, \omega\right) \chi_{m}\left(\mathbf{x}_{2}, \omega\right)+\right. \\
& \left.2 \chi_{e m}^{2}\left(\mathbf{x}_{2}, \omega\right)\right] \nabla_{1} \times \mathbf{G}^{0}\left(\mathbf{x}_{1}-\mathbf{x}_{2}, \omega\right) \nabla_{2} \times \mathbf{G}^{0}\left(\mathbf{x}_{2}-\mathbf{x}^{\prime}, \omega\right)+
\end{aligned}
$$

$$
\begin{aligned}
& +4 \int d^{3} \mathbf{x}_{2} \int d^{3} \mathbf{x}_{1} G^{0}\left(\mathbf{x}-\mathbf{x}_{1}, \omega\right)\left[i \omega^{3} \chi_{e}\left(\mathbf{x}_{1}, \omega\right) \chi_{e m}\left(\mathbf{x}_{2}, \omega\right)\right] \times \\
& \nabla_{1} \times \mathbf{G}^{0}\left(\mathbf{x}_{1}-\mathbf{x}_{2}, \omega\right) \mathbf{G}^{0}\left(\mathbf{x}_{2}-\mathbf{x}^{\prime}, \omega\right)+ \\
& \left.4 \int d^{3} \mathbf{x}_{2} \int d^{3} \mathbf{x}_{1} \nabla_{1} \times \mathbf{G}^{0}\left(\mathbf{x}-\mathbf{x}_{1}, \omega\right)\left[i \omega \chi_{m}\left(\mathbf{x}_{1}, \omega\right) \chi_{e m}\left(\mathbf{x}_{2}, \omega\right)\right)\right] \times \\
& \nabla_{1} \times \nabla_{2} \times \mathbf{G}^{0}\left(\mathbf{x}_{1}-\mathbf{x}_{2}, \omega\right) \mathbf{G}^{0}\left(\mathbf{x}_{2}-\mathbf{x}^{\prime}, \omega\right)+\cdots
\end{aligned}
$$

where, $\chi_{i}(\omega, \mathbf{x})$ are susceptibility functions of the medium and in terms of coupling function of electromagnetic field and bi-isotropic materials are as follows:

$$
\begin{aligned}
& \chi_{e}(\mathbf{x}, \omega)=\frac{1}{\varepsilon_{0}} \int d \omega^{\prime} \frac{f_{1}^{2}\left(\mathbf{x}, \omega^{\prime}\right)+f_{2}^{2}\left(\mathbf{x}, \omega^{\prime}\right)}{\omega^{\prime 2}-\omega^{2}-i 0^{+}} \\
& \chi_{m}(\mathbf{x}, \omega)=\mu_{0} \int d \omega^{\prime} \frac{g_{1}^{2}\left(\mathbf{x}, \omega^{\prime}\right)+g_{2}^{2}\left(\mathbf{x}, \omega^{\prime}\right)}{\omega^{\prime 2}-\omega^{2}-i 0^{+}} \\
& \chi_{e m}(\mathbf{x}, \omega)= \\
& \int d \omega^{\prime} \frac{f_{1}\left(\mathbf{x}, \omega^{\prime}\right) g_{1}\left(\mathbf{x}, \omega^{\prime}\right)+f_{2}\left(\mathbf{x}, \omega^{\prime}\right) g_{2}\left(\mathbf{x}, \omega^{\prime}\right)}{\omega^{\prime 2}-\omega^{2}-i 0^{+}}
\end{aligned}
$$

The partition function of electromagnetic field in the presence of the medium is $\Xi=\int D[\mathbf{A}] \exp \left(\frac{i}{\hbar} \int d^{4} x L\right)$, where $L$ is given by Eq. (1).

If we make a wick's rotation $\omega=i v_{l}$ in frequency space, the action will be Euclidean and the free energy is given by $\mathrm{E}=-\hbar / \tau \ln \Xi$, where $\tau$ is the duration of the interaction. After some straightforward calculation, the free energy is obtained in terms of green's function of interacting electromagnetic field:

$$
\mathrm{E}=-k_{B} T \sum_{l=0}^{\infty} \operatorname{tr} \ln \left[\mathbf{G}_{E M-b i}\left(\mathbf{x}-\mathbf{x}^{\prime}, \omega=i v_{l}\right)\right]
$$

where $v_{l}=2 \pi l k_{B} T / \hbar$ are Matsubara frequencies and $K_{B}$ is Boltzmann's constant.

\section{QUANTUM DYNAMICS}

To show the quantum treatment of electromagnetic field in the presence of biisotropic metamaterials, we start from the lagrangian of the system. Using EulerLagrange equation, the equation of motion of fields $\mathbf{A}, \mathbf{X}_{\omega}$, and $\mathbf{Y}_{\omega}$ are obtained as below: 


$$
\begin{aligned}
& \frac{1}{\mu_{0}} \nabla \times \nabla \times \mathbf{A}(\mathbf{x}, \mathrm{t})+\varepsilon_{0} \frac{\partial^{2} \mathbf{A}(\mathbf{x}, \mathrm{t})}{\partial t^{2}}= \\
& \nabla \times\left[\mathrm{g}_{1}(\mathbf{x}, \omega) \mathbf{X}_{\omega}(\mathbf{x}, \mathrm{t})\right]+\nabla \times\left[\mathrm{g}_{2}(\mathbf{x}, \omega) \mathbf{Y}_{\omega}(\mathbf{x}, \mathrm{t})\right]- \\
& \mathrm{f}_{1}(\mathbf{x}, \omega) \dot{\mathbf{X}}_{\omega}(\mathbf{x}, \mathrm{t})-\mathrm{f}_{2}(\mathbf{x}, \omega) \dot{\mathbf{Y}}_{\omega}(\mathbf{x}, \mathrm{t}) \\
& \ddot{\mathbf{X}}_{\omega}(\mathbf{x}, \mathrm{t})+\omega^{2} \mathbf{X}_{\omega}=f_{1}(\mathbf{x}, \omega) \dot{\mathbf{A}}(\mathbf{x}, \mathrm{t})+ \\
& g_{1}(\mathbf{x}, \omega) \nabla \times \mathbf{A}(\mathbf{x}, \mathrm{t}) \\
& \ddot{\mathbf{Y}}_{\omega}(\mathbf{x}, \mathrm{t})+\omega^{2} \mathbf{Y}_{\omega}=f_{2}(\mathbf{x}, \omega) \dot{\mathbf{A}}(\mathbf{x}, \mathrm{t})+ \\
& g_{2}(\mathbf{x}, \omega) \nabla \times \mathbf{A}(\mathbf{x}, \mathrm{t})
\end{aligned}
$$

The formal solution of the field Eqs. $(13,14)$ are

$$
\begin{gathered}
\mathbf{X}_{\omega}(\mathbf{x}, \mathrm{t})=\mathbf{X}_{\omega}(\mathbf{x}, 0) \cos \omega \mathrm{t}+\frac{\sin \omega t}{\omega} \dot{\mathbf{X}}_{\omega}(\mathbf{x}, 0) \\
+\int_{0}^{t} d t^{\prime} \frac{\sin \omega\left(\mathrm{t}-\mathrm{t}^{\prime}\right)}{\omega}\left[f_{1}(\mathbf{x}, \omega) \dot{\mathbf{A}}(\mathbf{x}, \mathrm{t})+\right. \\
\left.g_{1}(\mathbf{x}, \omega) \nabla \times \mathbf{A}(\mathbf{x}, \mathrm{t})\right]
\end{gathered}
$$

and

$$
\begin{aligned}
\mathbf{Y}_{\omega}(\mathbf{x}, \mathrm{t}) & =\mathbf{Y}_{\omega}(\mathbf{x}, 0) \cos \omega \mathrm{t}+\frac{\sin \omega t}{\omega} \dot{\mathbf{Y}}_{\omega}(\mathbf{x}, 0)+ \\
& \int_{0}^{t} d t^{\prime} \frac{\sin \omega\left(\mathrm{t}-\mathrm{t}^{\prime}\right)}{\omega}\left[f_{2}(\mathbf{x}, \omega) \dot{\mathbf{A}}(\mathbf{x}, \mathrm{t})+\right. \\
& \left.g_{2}(\mathbf{x}, \omega) \nabla \times \mathbf{A}(\mathbf{x}, \mathrm{t})\right]
\end{aligned}
$$

In this equation, the first term is the homogeneous solution of Eqs. (15) and (16) and the second term is the inhomogeneous one. By substituting Eqs. (15) and (16) into Eq. (12), the Langevin equation is obtained as

$$
\begin{aligned}
& {\left[\nabla \times\left(1-\chi_{m}(\mathbf{x}, \omega)\right) \nabla \times \mathbf{A}(\mathbf{x}, \omega)\right]} \\
& -\frac{\omega^{2}}{c^{2}}\left[1+\chi_{e}(\mathbf{x}, \omega)\right] \mathbf{A}(\mathbf{x}, \omega)-i \omega \mu_{0} \nabla \chi_{e m}(\mathbf{x}, \omega) \times \mathbf{A}(\mathbf{x}, \omega) \\
& =\mu_{0} \nabla \times \mathbf{M}_{N}(\mathbf{x}, \omega)-i \omega \mu_{0} \mathbf{P}_{N}(\mathbf{x}, \omega)
\end{aligned}
$$

where $\mathbf{P}_{N}(\mathbf{x}, \mathrm{t})$ and $\mathbf{M}_{N}(\mathbf{x}, \mathrm{t})$ are the electric and magnetic polarization noise densities associated with absorption, with the causal behavior of the medium respectively.

$$
\begin{aligned}
& \mathbf{P}_{N}(\mathbf{x}, \mathrm{t})= \\
& \int_{0}^{\infty} d \omega\left\{\mathrm{f}_{1}(\mathbf{x}, \omega)\left[\mathbf{X}_{\omega}(\mathbf{x}, 0) \cos \omega \mathrm{t}+\frac{\sin \omega t}{\omega} \dot{\mathbf{X}}_{\omega}(\mathbf{x}, 0)\right]+\right. \\
& \left.\mathrm{f}_{2}(\mathbf{x}, \omega)\left[\mathbf{Y}_{\omega}(\mathbf{x}, 0) \cos \omega \mathrm{t}+\frac{\sin \omega t}{\omega} \dot{\mathbf{Y}}_{\omega}(\mathbf{x}, 0)\right]\right\}
\end{aligned}
$$

and

$$
\begin{aligned}
& \mathbf{M}_{N}(\mathbf{x}, \mathrm{t})= \\
& \int_{0}^{\infty} d \omega\left\{\mathrm{g}_{1}(\mathbf{x}, \omega)\left[\mathbf{X}_{\omega}(\mathbf{x}, 0) \cos \omega \mathrm{t}+\frac{\sin \omega t}{\omega} \dot{\mathbf{X}}_{\omega}(\mathbf{x}, 0)\right]\right. \\
& \left.\quad+g_{2}(\mathbf{x}, \omega)\left[\mathbf{Y}_{\omega}(\mathbf{x}, 0) \cos \omega \mathrm{t}+\frac{\sin \omega t}{\omega} \dot{\mathbf{Y}}_{\omega}(\mathbf{x}, 0)\right]\right\}
\end{aligned}
$$

We can show that the Green's function of the interacting electromagnetic field satisfies

$$
\begin{aligned}
& \nabla \times\left[1-\chi_{m}(\mathbf{x}, \omega)\right] \nabla \times \mathbf{G}\left(\mathbf{x}-\mathbf{x}^{\prime}, \omega\right)- \\
& \frac{\omega^{2}}{c^{2}}\left[\left[1+\chi_{e}(\mathbf{x}, \omega)\right]\right] \mathbf{G}\left(\mathbf{x}-\mathbf{x}^{\prime}, \omega\right)- \\
& i \omega \mu_{0} \nabla \chi_{e m}(\mathbf{x}, \omega) \times \mathbf{G}\left(\mathbf{x}-\mathbf{x}^{\prime}, \omega\right)=\mu_{0} \delta\left(\mathbf{x}-\mathbf{x}^{\prime}\right) \mathbf{1}_{0}
\end{aligned}
$$

The two point correlation function Eq. (9) satisfied Eq. (20).

\section{CASIMIr ENERgy OF MetAMATERIALS}

In this section we consider two applications of the relations which were obtained in the previous section.In section 4-1, two polarizable isotropic medium in the presence of the electromagnetic fields is investigated. In section 4-2, Casimir force of two polarizable and magnetizable medium is calculated.

\section{A. Casimir Force of Polarizable Metamaterials}

We consider an isotropic polarizable metamaterial in the presence of electromagnetic field that is a special case of Eq. (4), so $g_{1}(\mathbf{x}, \omega)=g_{2}(\mathbf{x}, \omega)=f_{2}(\mathbf{x}, \omega)=0$. The expansion of free energy in terms of electrical susceptibility function is obtained using Eqs. $(9,11)$, 


$$
\begin{aligned}
E= & K_{B} T \sum_{l=0}^{\infty} \sum_{n=1}^{\infty} \frac{(-1)^{n+1}}{n} \int d^{3} \mathbf{x}_{1} \cdots d^{3} \mathbf{x}_{n} \times \\
& \mathbf{G}_{i_{1, i}}^{0}\left(i v_{l} ; \mathbf{x}_{1}-\mathbf{x}_{2}\right) \ldots \mathbf{G}_{i_{n} i_{1}}^{0}\left(i v_{l} ; \mathbf{x}_{n}-\mathbf{x}_{1}\right) \times \\
& \chi_{e}\left(i v_{l}, \mathbf{x}_{1}\right) \cdots \chi_{e}\left(i v_{l}, \mathbf{x}_{n}\right)
\end{aligned}
$$

where in second approximation is

$$
\begin{aligned}
E= & -\frac{1}{2} K_{B} T \sum_{l=0}^{\infty} \iint_{V_{1} V_{2}} d^{3} \mathbf{x} d^{3} \mathbf{x}^{\prime} \times \\
& \mathbf{G}_{i j}^{0}\left(\mathbf{x}-\mathbf{x}^{\prime}, i v_{l}\right) \mathbf{G}_{j i}^{0}\left(\mathbf{x}^{\prime}-\mathbf{x}, i v_{l}\right) \times \\
& \chi_{e 1}\left(i v_{l}, \mathbf{x}\right) \chi_{e 2}\left(i v_{l}, \mathbf{x}^{\prime}\right)
\end{aligned}
$$

At zero temperature, the summation over the positive integer 1 is replaced by an integral. For example, we calculate the free energy of the electromagnetic field in the presence of two nanospheres with radii of $a$ and $b$, the distance between their centers $R>a+b$ with susceptibilities $\quad \chi_{e 1}(\mathbf{x}, \omega)=\chi_{e 1}(\omega) \delta(\mathbf{r}-\mathbf{a}) \quad$ and $\chi_{e 2}\left(\mathbf{x}^{\prime}, \omega\right)=\chi_{e 2}(\omega) \delta\left(\mathbf{r}^{\prime}-\mathbf{b}\right)$, where $\mathbf{r}$ and $\mathbf{r}^{\prime}$ are radial coordinates in spherical coordinate system. In this section, homogeneoussingleresonance Lorentz-oscillator models is chosen for materials. Thus, electric response is $\chi_{e}(\mathrm{i} \omega)=\frac{\omega_{p e}^{2}}{\omega_{0 e}^{2}+\omega^{2}+\gamma_{e} \omega}$, where $\omega_{p}, \omega_{0}$ and $\gamma$ are coupling frequency, transverse resonance frequency and damping coefficient, respectively. It is shown in Fig. 1 that the electrialc part of thepermittivity is negative for frequencies $\omega_{0 e}<\omega<\sqrt{\omega_{0 e}^{2}+\omega_{p e}^{2}}$ and the material acts as a metamaterial in this frequency.

After some straightforward calculation of free energy of the system and assuming $R>>(a+b)$, the Casimir force is obtained as follows:

$$
\begin{aligned}
F & =\frac{-\hbar a^{2} b^{2}}{\pi} \int d v\left(\frac{\omega_{p e}^{2}}{\omega_{0 e}^{2}+v^{2}+\gamma_{e} v}\right) \\
& \times\left(\frac{\omega_{p e}^{\prime 2}}{\omega_{0 e}^{\prime 2}+v^{2}+\gamma_{e} v}\right) \exp \left(-\frac{2 v R}{c}\right)\left[\frac{2 v^{5}}{c^{5} R^{2}}+\frac{6 v^{4}}{c^{4} R^{3}}\right. \\
& \left.+\frac{16 v^{3}}{c^{3} R^{4}}+\frac{32 v^{2}}{c^{2} R^{5}}+\frac{36 v}{c R^{6}}+\frac{18}{R^{7}}\right]
\end{aligned}
$$


example, the magnetic permeability is exerted in the calculation.

\section{B. Casimir Force in Polarizable- Magnetizable Metamaterials}

In this section, the magnetic permeability is exerted in the calculation. We consider two polarizable and magnetizable medium, so in Eq. (4), $\quad g_{1}(\mathbf{x}, \omega)=f_{2}(\mathbf{x}, \omega)=0$. The system composed of two bodies with volumes $V_{1}$ and $V_{2}$ and the susceptibilities $\chi_{e}$ and $\chi_{m}$. The first relevant nonzero term for the free energy of the system is

$$
\begin{array}{r}
E=-k_{B} T \sum_{l=0}^{\infty} \int_{V_{1}} \int_{V_{2}} d^{3} \mathbf{x}_{1} d^{3} \mathbf{x}_{2} \nabla_{1} \times \mathbf{G}_{i j}^{0}\left(\mathbf{x}_{1}-\mathbf{x}_{2}, i v_{l}\right) \\
{\left[\chi_{e}\left(i v_{l}, \mathbf{x}_{1}\right) \chi_{m}\left(i v_{l}, \mathbf{x}_{2}\right)\right] \nabla_{2} \times \mathbf{G}_{j i}^{0}\left(\mathbf{x}_{2}-\mathbf{x}_{1}, i v_{l}\right)}
\end{array}
$$

By inserting the Green's function Eq. (9) in to Eq. (24), we find that:

$$
\begin{aligned}
E= & -k_{B} T \sum_{l=0}^{\infty} \int_{V_{1}} \int_{V_{2}} d^{3} \mathbf{x}_{1} d^{3} \mathbf{x}_{2} \\
& \chi_{e}\left(i v_{l}, \mathbf{x}_{1}\right) \chi_{m}\left(i v_{l}, \mathbf{x}_{2}\right) \times \mathrm{h}_{e m}\left(v_{l},\left|\mathbf{x}_{1}-\mathbf{x}_{2}\right|\right)
\end{aligned}
$$

where we have defined

$$
\begin{aligned}
& \mathrm{h}_{e m}\left(v_{l}, r\right)= \\
& \frac{\exp \left(-\frac{2 v_{l}}{c} r\right)}{8 \pi^{2}}\left\{\frac{\left(\frac{v_{l}}{c}\right)^{3}}{r}+\frac{2\left(\frac{v_{l}}{c}\right)^{2}}{r^{2}}+\frac{3\left(\frac{v_{l}}{c}\right)}{r^{3}}+\frac{3}{r^{4}}\right\}^{2}
\end{aligned}
$$

where $r=\left|\mathbf{x}_{1}-\mathbf{x}_{2}\right|$. After some straightforward calculation, the free energy is obtained as below:

$$
E=-\frac{333 \hbar}{128 \pi^{3}} \int d^{3} \mathbf{x}_{1} \int d^{3} \mathbf{x}_{2} \frac{\chi_{e}\left(\mathbf{x}_{1}\right) \chi_{m}\left(\mathbf{x}_{2}\right)}{\left|\mathbf{x}_{1}-\mathbf{x}_{2}\right|^{8}}
$$

The free energy for two spheres with radii of $a$ and $b$, the distance between their centers $R>a+b \quad$ with susceptibilities $\chi_{e}\left(\mathbf{x}_{1}\right)=\chi_{e} \delta(\mathbf{r}-\mathbf{a}) \quad$ and $\quad \chi_{m}\left(\mathbf{x}_{2}\right)=\chi_{m} \delta\left(\mathbf{r}^{\prime}-\mathbf{b}\right)$, where $\mathbf{r}$ and $\mathbf{r}^{\prime}$ are radial coordinates in spherical coordinate systems, is as follows

$$
E=-\frac{333 \hbar \chi_{e} \chi_{m} a^{2} b^{2}}{8 \pi R^{8}} P_{-8}(\hat{a}, \hat{b})
$$

where $\hat{a}=a / R, \hat{b}=b / R$ and

$$
\begin{aligned}
P_{-8}(\hat{a}, \hat{b})= & \frac{1}{10 \hat{a} \hat{b}}\left[\frac{1}{(\hat{a}-\hat{b}-1)^{5}}-\frac{1}{(\hat{a}+\hat{b}-1)^{5}}\right. \\
& \left.-\frac{1}{(\hat{a}-\hat{b}+1)^{5}}+\frac{1}{(\hat{a}+\hat{b}+1)^{5}}\right]
\end{aligned}
$$

The Casimir force is as follows

$$
\begin{aligned}
F= & -\frac{333 \hbar \chi_{e} \chi_{m} a^{2} b^{2}}{8 \pi R^{9}} P_{-8}(\hat{a}, \hat{b})- \\
& \frac{333 \times 7 \hbar \chi_{e} \chi_{m} a^{2} b^{2}}{8 \pi R^{9}} P_{-9}(\hat{a}, \hat{b})
\end{aligned}
$$

where

$$
\begin{aligned}
& P_{-9}(\hat{a}, \hat{b})=\frac{1}{14 \hat{a} \hat{b}}\left[\frac{-1}{(\hat{a}-\hat{b}-1)^{6}}+\right. \\
& \left.\frac{1}{(\hat{a}+\hat{b}-1)^{6}}-\frac{1}{(\hat{a}-\hat{b}+1)^{6}}+\frac{1}{(\hat{a}+\hat{b}+1)^{6}}\right]
\end{aligned}
$$

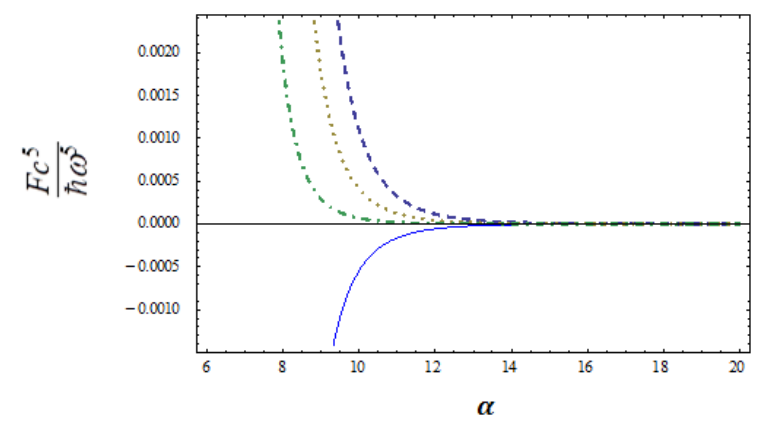

Fig. 3: Casimir force of two nanospheres as a function of distance between sphere centers, R. Parameters are: $a=2 \lambda, b=3 \lambda, R=\alpha \lambda>a+b$, where $\alpha$ is 6 from 20, $\varepsilon=\mu=-1$ (dashed), $\varepsilon=2, \mu=-1$ (blue), $\varepsilon=1 / 2, \mu=-1 / 2$ (dotted), $\varepsilon=1 / 2, \mu=2$ (dot-dashed).

In Fig. 3, we compare the Casimir force of two nanospheres as described by Eq. (30) for homogeneous media, all as a function of distance $\mathrm{R}$ for different electric permittivity and magnetic permeability. In the case of 
$\varepsilon=\mu=-1$, electric permittivity and magnetic permeability are near to the resonance frequency of the metamaterial, so the Casimir force is repulsive. In the case $\varepsilon=1 / 2$ and $\mu=-1 / 2$, the Casimir force is repulsive due to negative magnetic permeability. In the case $\varepsilon=2$ and $\mu=-1$, the Casimir force is attractive, despite magnetic permeability is negative. The reason may be due to strong electric permittivity. In the case $\varepsilon=1 / 2$ and $\mu=2$, the Casimir force is repulsive, despite positive magnetic permeability and electric permittivity. It is attributed to the strong magnetic permeability which is shown in metamaterials.

\section{CONCLUSION}

The present research investigated the quantum field theory of bi-isotropic metamaterial in the presence of electromagnetic fields. Using generating functional, two-point correlation function is obtained. Two-point correlation function or Green's function is a series expansion in terms of the susceptibility function of the medium. An illustrative example is given, showing the applicability of the method. Using two-point Green's function, the free energy of the system was obtained and the Casimir energy of polarizable and magnetizable-polarizable metamaterials were studied. It was shown that the Casimir force of two polarizable nanospheres was attractive for all frequencies. By exerting magnetic permeability into calculation, in some cases, the repulsive casimir force between two nanospheres was revealed.

\section{REFERENCES}

[1] W. Greiner and J. Reinhardt, Quantum Electrodynamics, Springer-Verlag Berlin, Heidelberg, $4^{\text {th }}$ Ed. 2009.

[2] H. Kleinert, Path Integrals in Quantum Mechanics, Statistics, Polymer Physics, and Financial Markets, World Scientic, Singapore, $5^{\text {th }}$ Ed. 2009.

[3] W. Vogel and D. G. Welsch, Quantum Optics, Wiley- VCH Verlag $\mathrm{GmbH}$ and Co. Weinheim, $3^{\text {rd }}$ Ed. 2006.
[4] M. Amooshahi, "Canonical quantization of electromagnetic field in the presence of absorbing bi-anisotropic multilayer magnetodielectric media," Eur. Phys. J. D, Vol. 69, PP. 66-88, 2015.

[5] R. Nawaz and M. Ayub, "Closed form solution of electromagnetic wave diffraction problem in a homogeneous bi-isotropic medium," Math. Method Appl. Sci. Vol. 38, PP. 176-187, 2016.

[6] M.A. Kaliteevski, V.A. Mazlin, K. A. Ivanov, and A. R. Gubaydullin, "Quantization of electromagnetic field in an inhomogeneous medium based on scattering matrix formalism (S-quantization)", Opt. Spectrosc. Vol. 119, PP. 832-837, 2015.

[7] S.A.R. Horsley, "Canonical quantization of the electromagnetic field interacting with a moving dielectric medium," Phys. Rev. A, Vol. 86, PP. 023830 (1-14), 2012.

[8] F. Kheirandish and S. Salimi, "Quantum field theory in the presence of a medium: Green's function expansions," Phys. Rev. A, Vol. 84, PP. 062122 (1-9), 2011.

[9] M. F. Maghrebi, R. Golestanian, and M. Kardar, "Scattering approach to the dynamical Casimir effect," Physical Review D, Vol. 87, PP. 025016 (1-15), 2013.

[10] F. Kheirandish and M. Jafar "A perturbative approach to calculating the Casimir force in fluctuating scalar and vector fields," Phys.Rev. A, Vol. 86, PP. 022503 (1-20), 2012.

[11] R. Golestanian and M. Kardar, "Path-integral approach to the dynamic Casimir effect with fluctuating boundaries," Physical Review A, Vol. 58, PP. 1713-1722, 1998.

[12] B. Kiani and J. Sarabadani, "Repulsive Casimir interaction between conducting and permeable corrugated plates," Phys. Rev. A, Vol. 86, PP. 022516 (1-6), 2012.

[13] R. Guerout, A. Lambrecht, K. A. Milton, and S Reynaud, "Lifshitz-Matsubara sum formula for the Casimir pressure between magnetic metallic mirrors," Phys. Rev. E, Vol. 93, PP. 022108 (1-7), 2016.

[14] J S. Høye, I. Brevik, and K. A. Milton, "Casimir friction between polarizable particle and half-space with radiation damping at zero temperature," J. Phys. A: Math. Theor. Vol. 84, PP. 36-51, 2016. 
[15] E. Amooghorban and F. Kheirandish, "Relativistic and Non-Relativistic Quantum Brownian Motion in an Anisotropic Dissipative Medium," Int. J. Theor. Phys. Vol. 53, PP. 2593 (1-27), 2014.

[16] A. Refaei and F. Kheirandish, "Dissipative Scalar Field Theory: A Covariant Formulation," Int. J. Theor. Phys. Vol. 55, PP. 432-439, 2016.

[17] F. Kheirandish and V. Ameri, "Electromagnetic field quantization in the presence of a rotating body," Phys. Rev. A, Vol. 89, PP. 032124 (1-14), 2014.

[18] M. Amooshahi, "Canonical quantization of electromagnetic field in an anisotropic polarizable and magnetizable medium," J. Math. Phys, Vol. 50, PP. 062301 (1-22), 2009.

[19] W. Greiner and J. Richardt, Field quantization, Springer-Verlag, Berlin, Heidelberg, 1996.

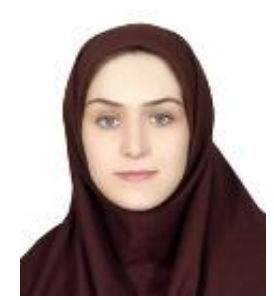

Marjan Jafari was born in Khorramdareh, Zanjan, on September 11, 1984. She received her B.Sc. in Solid State Physics in 2006. She also received her M.Sc. and Ph.D. degrees in Theoretical Physics from Physics Department of Isfahan University, Isfahan, Iran, under supervision of Professor Kheirandish, in 2009 and 2013, respectively. Dr Jafari research interests are Quantum Information, Quantum Optics, Quantum Field Theory, Casimir Effect, and Nanoelectromechanics.



Fateme Moradi was born in Qazvin in Jan. 2, 1992. She received her B.Sc in Solid State Physics from Physics Department of Zanjan University, Zanjan, Iran in, 2014 and her M.Sc. in atomic and molecular physics -optics under supervision of Ms. Dr. M. Jafari, from Physics Department of Imam Khomeini International University, Qazvin, Iran in 2017. 